\title{
Solubility of Benzodiazepines in Polyethylene Glycol $200+$ Water Mixtures at 303.2 K
}

\author{
Abolghasem Jouyban, ${ }^{, \dagger}$ Javad Shokri, ${ }^{\ddagger}$ Mohammad Barzegar-Jalali, ${ }^{\ddagger}$ Davoud Hassanzadeh, ${ }^{\S}$ \\ William E. Acree, Jr.," Taravat Ghafourian, ${ }^{\perp}$ and Ali Nokhodchi ${ }^{\perp}$
}

Faculty of Pharmacy and Drug Applied Research Center, Biotechnology Research Center, and Research Center for Pharmaceutical Nanotechnology, Tabriz University (Medical Sciences), Tabriz 51664, Iran, Department of Chemistry, University of North Texas, Denton, Texas 76203-5070, and Medway School of Pharmacy, Universities of Kent and Greenwich, Kent ME4 4TB, United Kingdom

\begin{abstract}
Experimental solubilities of chlordiazepoxide, diazepam, and lorazepam in polyethylene glycol 200 (PEG $200)+$ water mixtures at $303.2 \mathrm{~K}$ were reported. The solubility of each drug increased exponentially with the addition of PEG 200 and reached the maximum value in neat PEG 200. The Jouyban-Acree model was used to mathematically describe the experimental data, and the solubilities were predicted using a previously trained version of the Jouyban-Acree model for PEG + water mixtures and the solubility data in monosolvents. The overall mean relative deviations of the models were $3.7 \%$ and $18.3 \%$, respectively, for the fitted model and the trained version.
\end{abstract}

\section{Introduction}

Polyethylene glycols (PEGs) are water-soluble polymers with a general formula of $\mathrm{HO}-\left(\mathrm{CH}_{2} \mathrm{CH}_{2} \mathrm{O}\right)_{n}-\mathrm{H}$ and have been used in many pharmaceutical formulations including oral, parenteral, topical, ophthalmic, and rectal preparations. ${ }^{1,2}$ Generally, they are regarded as nontoxic and nonirritant materials. PEGs are also utilized in the industry to precipitate/crystallize proteins, and stable formulation of protein powders has been developed employing PEG-induced precipitation. ${ }^{3}$ PEGs are used in pharmaceutical formulations by acting as a cosolvent (lower molecular weight PEGs) to increase the aqueous solubility of poorly water-soluble drugs in liquid pharmaceutical formulations and/or as dissolution rate enhancers (higher molecular weight PEGs) in solid dispersions. ${ }^{4}$ PEGs are stable and low toxic pharmaceutical excipients which are used in many commercially available oral and parenteral pharmaceutical formulations of poorly soluble drugs. ${ }^{5,6}$ In addition to the solubilization power of polyethylene glycol 200 (PEG 200), it possesses stabilization ability on the enzymes and facilitates the biotransformation of low aqueous soluble substrates in aqueous solutions. ${ }^{7}$

The solubility of drugs in PEG $200+$ water mixtures is essential information in drug development studies. The composition of PEG 200 in pharmaceutical preparations should be kept as low as possible (usually a volume fraction less than $50 \%$ ), and the often used method to optimize the solvent composition of the mixtures for dissolving a desired amount of a drug in a given volume of the solution is the trial-and-error approach which is both timeconsuming and expensive. Moreover, in the early stages of drug discovery processes, the scarcity of the drug and/or drug candidate

\footnotetext{
* To whom correspondence should be addressed. E-mail: ajouyban@ hotmail.com. Fax: +98 4113363231.

$\uparrow$ Faculty of Pharmacy and Drug Applied Research Center, Tabriz University (Medical Sciences).

* Biotechnology Research Center, Tabriz University (Medical Sciences).

${ }^{\S}$ Research Center for Pharmaceutical Nanotechnology, Tabriz University (Medical Sciences).

"University of North Texas.

${ }^{\perp}$ Medway School of Pharmacy, Universities of Kent and Greenwich.
}

is another limiting factor. To address this issue, numerous mathematical models have been presented for predicting the solubility of drugs in water-cosolvent mixtures. These models and their advantages and limitations were recently reviewed. ${ }^{8}$

Of the models developed in recent years, the Jouyban-Acree model is perhaps one of the more versatile models. The model provides very accurate mathematical descriptions for how the solute solubility varies with both temperature and solvent composition. The model is

$$
\begin{aligned}
\log x_{\mathrm{m}, T}^{\mathrm{Sat}}=\varphi_{1} \log x_{1, T}^{\mathrm{Sat}}+\varphi_{2} \log x_{2, T}^{\mathrm{Sat}}+ & \\
& \frac{\varphi_{1} \varphi_{2}}{T / \mathrm{K}} \sum_{i=0}^{2} J_{i}\left(\varphi_{1}-\varphi_{2}\right)^{i}
\end{aligned}
$$

where $x_{\mathrm{m}, T}^{\mathrm{Sat}}$ is the solute (mole fraction) solubility in the binary solvent mixtures at temperature $T ; \varphi_{1}$ and $\varphi_{2}$ are the volume fractions of the solvents 1 (PEG 200) and 2 (water) in the absence of the solute; $x_{1, T}^{\text {Sat }}$ and $x_{2, T}^{\text {Sat }}$ denote the mole fraction solubility of the solute in solvents 1 and 2, respectively; and $J_{i}$ are the constants of the model computed by a regression analysis. ${ }^{8}$ Knowledge of these model constants, which requires a number of solubility data in water-cosolvent mixtures for the training process, is a limitation for the model when the solubility predictions are the goal of the computations in early drug discovery studies. This limitation could be overcome using a trained version of the model for a given water-cosolvent mixture. The trained version of the Jouyban-Acree model for prediction of drug solubility in PEG $400+$ water mixtures at temperature $(T)$ is 9

$$
\begin{array}{r}
\log x_{\mathrm{m}, T}^{\mathrm{Sat}}=\varphi_{1} \log x_{1, T}^{\mathrm{Sat}}+\varphi_{2} \log x_{2, T}^{\mathrm{Sat}}+\frac{394.82 \varphi_{1} \varphi_{2}}{T / \mathrm{K}}- \\
\frac{355.28 \varphi_{1} \varphi_{2}\left(\varphi_{1}-\varphi_{2}\right)}{T / \mathrm{K}}+\frac{388.89 \varphi_{1} \varphi_{2}\left(\varphi_{1}-\varphi_{2}\right)^{2}}{T / \mathrm{K}}
\end{array}
$$

which was trained using 80 data sets of Rytting et al. ${ }^{10}$ and produced reasonable prediction error $(\approx 40 \%) .{ }^{9}$ It should be noted that we considered that the addition of the solvent 1 


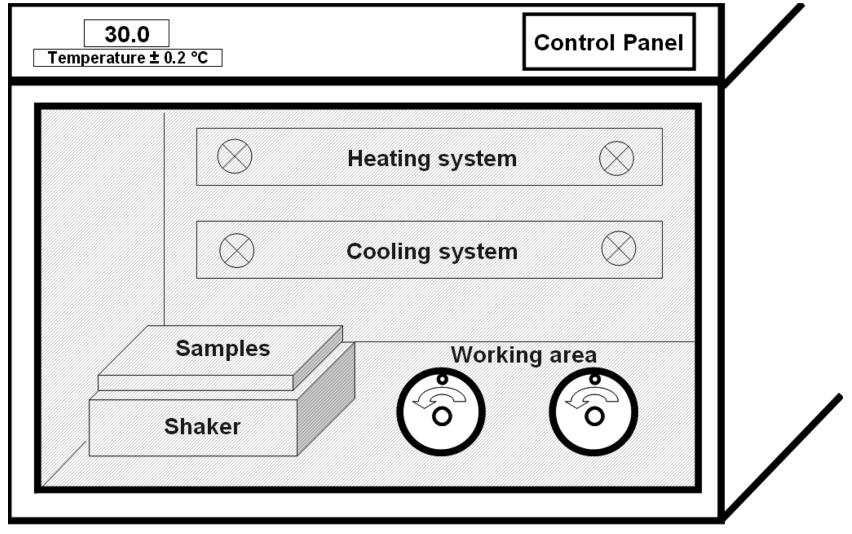

Figure 1. Schematic representation of the incubator used in the solubility determination procedure.

increases the solubility of drugs; in other words, the numerical value of solubility in solvent 1 is more than the solubility in solvent $2\left(x_{1, T}^{\text {Sat }}>x_{2, T}^{\text {Sat }}\right)$. Equation 2 was developed assuming that the extent of solute-solvent interactions in the binary solvent of PEG 400 and water are the same, and the effect of solute structures is only affected by $x_{1, T}^{\mathrm{Sat}}$ and $x_{2, T}^{\mathrm{Sat}}$ values. Since ethylene glycols possess the same functional groups, eq 2 is applicable for solubility prediction of drugs in aqueous mixtures of ethylene glycols (including PEG 200) as shown in a previous report. ${ }^{11}$

There were no published data on the solubility of chlordiazepoxide, diazepam, and lorazepam in PEG 200 + water mixtures at $303.2 \mathrm{~K}$. The aim of this work is to report the solubility of chlordiazepoxide, diazepam, and lorazepam in binary aqueous mixtures of PEG 200 at $303.2 \mathrm{~K}$. In addition, the applicability of the Jouyban-Acree model to the measured solubility data and the prediction capability of the above-mentioned trained model for predicting the solubility of these drugs in PEG 200 + water mixtures were investigated.

\section{Experimental Method}

Materials. Chlordiazepoxide (CAS \# 58-25-3), diazepam (CAS \# 439-14-5), and lorazepam (CAS \# 846-49-1) were purchased from Loghman pharmaceutical company (Iran). PEG $200(99.7 \%)$ was purchased from Merck (Germany); methanol $(99.8 \%)$ was obtained from Caledon (Canada); and double distilled water was used for preparation of the solutions.
Solubility Determinations. The binary solvent mixtures were prepared by mixing the appropriate volumes of the solvents with the uncertainty of 0.001 volume fraction. The solubility of the three benzodiazepines in PEG $200+$ water mixtures was determined by equilibrating excess amounts of the solids at 303.2 K using a shaker (Behdad, Tehran, Iran) placed in an incubator equipped with a temperature-controlling system maintained constant to within $\pm 0.2 \mathrm{~K}$ (see Figure 1). After a sufficient length of time ( $>24 \mathrm{~h}$ ), the saturated solutions were filtered using hydrophilic Durapore filters $(0.45 \mu \mathrm{m}$, Milipore, Ireland), and diluted with methanol. After the first dilution, the solutions are further diluted at the laboratory temperature and then assayed using a UV-vis spectrophotometer (Beckman DU650 , Fullerton). Concentrations of the diluted solutions were determined from calibration curves. Details of calibration curves are summarized in Table 1. Each experimental data point represents the average of at least three repetitive experiments with the measured mole fraction solubilities being reproducible on a relative basis to within $\pm 4.9 \%$. The relative standard deviations of mole fraction solubilities ranged from $0.6 \%$ to $5.0 \%$. The densities of the saturated solutions were determined using a $5 \mathrm{~mL}$ pycnometer with the uncertainty of $\left(\sigma_{n-1}=0.004\right.$ to $\left.\sigma_{n-1}=0.028\right) \mathrm{g} \cdot \mathrm{cm}^{-3}$.

Computational Methods. In the numerical analysis of method I, eq 1 was fitted to the experimental solubility data of each drug, and the back-calculated solubilities were used to calculate the accuracy of the fit. In method II, the solubilities of three drugs were predicted using eq 2 employing the experimental solubilities of drugs in neat PEG 200 and water. The mean relative deviation (MRD) was used to check the accuracy of each prediction method and is calculated using

$$
\operatorname{MRD}=\frac{1}{N} \sum_{i=1}^{N}\left\{\frac{\mathrm{I}\left(X_{\mathrm{m}}^{\mathrm{Sat}}\right)_{i}^{\text {Predicted }}-\left(X_{\mathrm{m}}^{\mathrm{Sat}}\right)_{i}^{\text {experimental }} \mathrm{I}}{\left(X_{\mathrm{m}}^{\mathrm{Sat}}\right)_{i}^{\text {experimental }}}\right\}
$$

where $N$ is the number of data points in each set. Goodnessof-fit to the method was also shown by plotting the predicted and experimental solubilities of the drugs against the volume fraction of PEG 200.

\section{Results and Discussion}

Table 2 lists the experimental solubilities of chlordiazepoxide, diazepam, and lorazepam in PEG 200 + water mixtures at 303.2

Table 1. Details of Calibration Curves of Drugs

\begin{tabular}{lccccc}
\hline \multicolumn{1}{c}{ drug } & $\lambda / \mathrm{nm}$ & $\varepsilon / \mathrm{L} \cdot \mathrm{mol}^{-1} \cdot \mathrm{cm}^{-1}$ & $c / \mathrm{mol} \cdot \mathrm{L}^{-1}$ & correlation coefficient & calibration curve $(A:$ absorbance $)$ \\
\hline chlordiazepoxide & 234 & 30167 to 31568 & $2.25 \cdot 10^{-6}$ to $3.34 \cdot 10^{-5}$ & 0.999 & $c=3.326 \cdot 10^{-5} A-1.125 \cdot 10^{-7}$ \\
diazepam & 231 & 33234 to 38033 & $2.37 \cdot 10^{-6}$ to $3.51 \cdot 10^{-5}$ & 0.999 & $c=3.041 \cdot 10^{-5} A-3.710 \cdot 10^{-7}$ \\
lorazepam & 231 & 37980 to 38924 & $2.10 \cdot 10^{-5}$ to $3.11 \cdot 10^{-5}$ & 0.999 & $c=2.638 \cdot 10^{-5} A-5.615 \cdot 10^{-8}$
\end{tabular}

Table 2. Experimental Mole Fraction Solubilities of Drugs $\left(x_{\mathrm{m}, T}^{\mathrm{Sat}}\right)$ in Different Volume Fractions of Polyethylene Glycol $200\left(\varphi_{1}\right)$ in Polyethylene Glycol 200 (1) + Water (2) Mixtures at 303.2 K and Density $\rho$ of the Saturated Solutions

\begin{tabular}{|c|c|c|c|c|c|c|}
\hline \multirow[b]{2}{*}{$\varphi_{1}$} & \multicolumn{3}{|c|}{$x_{\mathrm{m}, T}^{\mathrm{Sat}}$} & \multicolumn{3}{|c|}{$\rho / \mathrm{g} \cdot \mathrm{cm}^{-3}$} \\
\hline & chlordiazepoxide & diazepam & lorazepam & chlordiazepoxide & diazepam & lorazepam \\
\hline 0.000 & 0.000006 & 0.000004 & 0.000003 & 0.998 & 0.998 & 0.998 \\
\hline 0.100 & 0.000034 & 0.000017 & 0.000015 & 1.014 & 1.016 & 1.020 \\
\hline 0.200 & 0.000091 & 0.000044 & 0.000046 & 1.030 & 1.032 & 1.035 \\
\hline 0.300 & 0.000172 & 0.000100 & 0.000119 & 1.052 & 1.053 & 1.050 \\
\hline 0.400 & 0.000331 & 0.000233 & 0.000334 & 1.068 & 1.061 & 1.072 \\
\hline 0.500 & 0.000704 & 0.000502 & 0.000864 & 1.082 & 1.084 & 1.082 \\
\hline 0.600 & 0.001600 & 0.001230 & 0.002650 & 1.099 & 1.098 & 1.094 \\
\hline 0.700 & 0.002950 & 0.003330 & 0.008250 & 1.103 & 1.106 & 1.116 \\
\hline 0.800 & 0.006400 & 0.007290 & 0.025700 & 1.113 & 1.116 & 1.129 \\
\hline 0.900 & 0.013800 & 0.016850 & 0.057700 & 1.124 & 1.122 & 1.146 \\
\hline 1.000 & 0.023180 & 0.034380 & 0.095800 & 1.130 & 1.129 & 1.163 \\
\hline
\end{tabular}




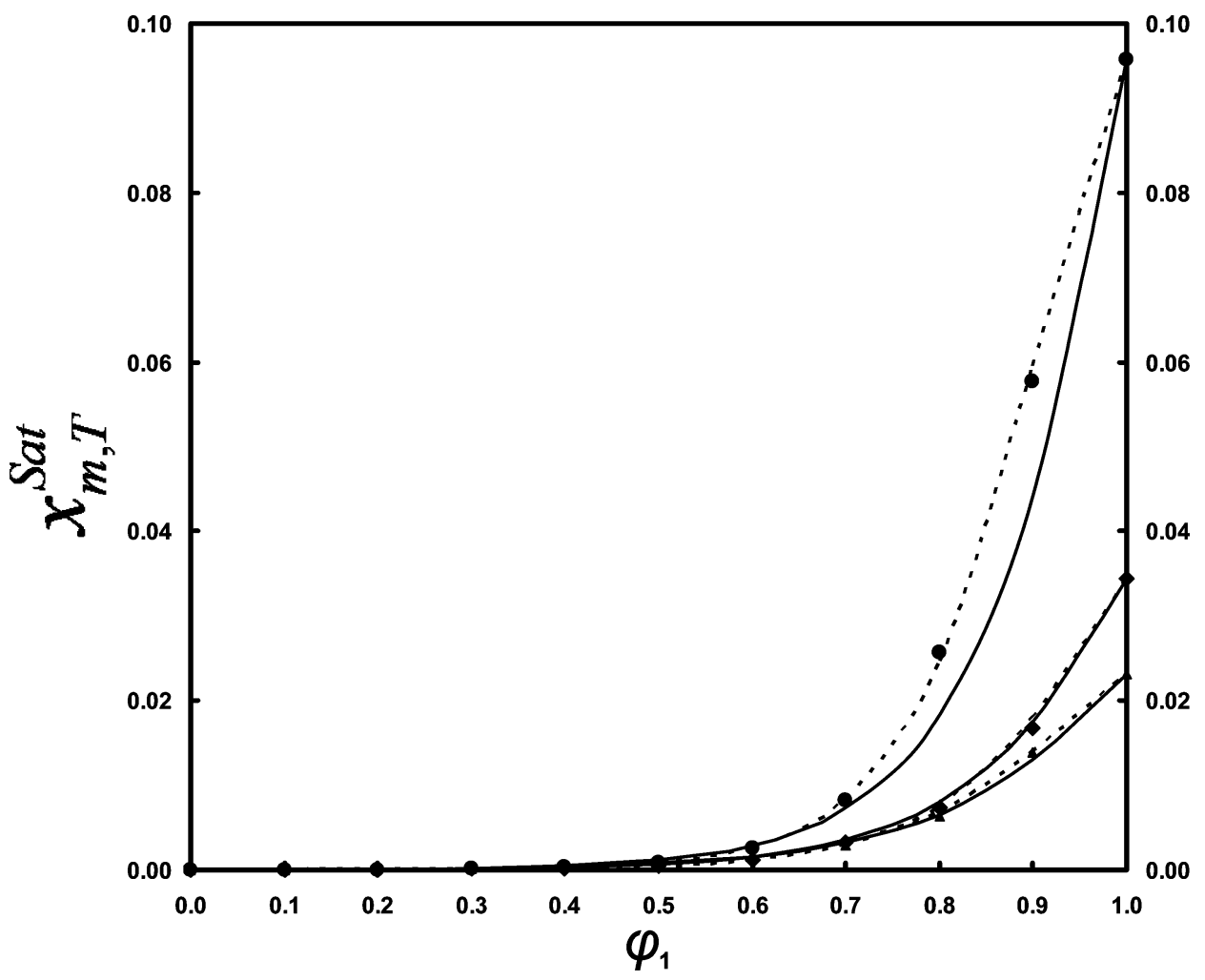

Figure 2. Experimental mole fraction solubility $\left(x_{\mathrm{m}, T}^{\mathrm{Sat}}\right)$ of $\boldsymbol{\Lambda}$, chlordiazepoxide; $\bullet$, diazepam; and $\boldsymbol{\bullet}$, and lorazepam at various volume fractions of polyethylene glycol $200\left(\varphi_{1}\right)$ in binary solvent mixtures and the corresponding computed solubilities using - - , method I; and -, method II.

K. Our measured value for the solubility of diazepam in water $\left(0.194 \mathrm{mmol} \cdot \mathrm{L}^{-1}\right.$ at $\left.303.2 \mathrm{~K}\right)$ is larger than the published solubility data of Loftsson and Hreinsdottir (0.14817 $\mathrm{mmol} \cdot \mathrm{L}^{-1}$ at $(295.15$ to 297.15$\left.) \mathrm{K}\right)^{12}$ and of Shyanfar et al. $\left(0.15 \mathrm{mmol} \cdot \mathrm{L}^{-1} \text { at } 298.15 \mathrm{~K}\right)^{13}$ as expected. Published studies were performed at slightly lower temperatures, and the solubility of crystalline materials increases with temperature as is evident from this work. The solubility of the benzodiazepines was predicted using numerical methods I and II. The experimental and predicted solubilities of the drugs versus the volume fraction of PEG 200 in the binary mixtures were plotted in Figure 2. As shown in the figure, the Jouyban-Acree model provides a very good mathematical description of the experimental solubility data of drugs at all composition ranges of PEG 200. The predicted data by using the previously trained eq 2 agree reasonably well with the corresponding experimental solubilities for chlordiazepoxide and diazepam and deviated slightly for lorazepam in the PEG 200 rich area. This finding is also supported by small MRD values between the backcalculated and experimental solubility data.

The main limitation of eq 1 is that it should be trained for each drug employing a minimum number of experimental data in binary solvents. The predictive version of the model, i.e., eq 2 , predicts the solubility values with reasonable MRD values. To the best of our knowledge, there is no accurate model to predict the solubility of drugs in monosolvents at various temperatures; therefore, the experimental values of $x_{1, T}^{\mathrm{Sat}}$ and $x_{2, T}^{\mathrm{Sat}}$ should be used as input data to predict the solubilities in mixed solvents. The predicted solubilities were compared with the corresponding experimental data, and MRD values were computed and listed in Table 3. Generally, the overall MRDs of the Jouyban-Acree model reveal that its trained version for PEG $400+$ water mixtures is a robust model to predict the
Table 3. Numerical Values of Adjusted Parameters of Equation 1 for Each Drug and the Mean Relative Deviation (MRD) for the Predicted Solubilities Using Various Numerical Methods and Their Overall Values

\begin{tabular}{lcrccc}
\hline \multicolumn{1}{c}{ drug } & $J_{0}$ & \multicolumn{1}{c}{$J_{1}$} & $J_{2}$ & method I & method II \\
\hline chlordiazepoxide & 320.53 & -428.60 & 792.36 & 5.5 & 8.7 \\
diazepam & 209.22 & -257.60 & 604.27 & 3.6 & 19.1 \\
lorazepam & 227.17 & 106.96 & 760.30 & 1.9 & 27.1 \\
& & & overall: & 3.7 & 18.3
\end{tabular}

solubility of drugs in PEG + water mixtures and could be used for prediction purposes with a relative uncertainty of less than $19 \%$.

\section{Literature Cited}

(1) Broadhead, J. Parentral Dosage Form. In Pharmaceutical Preformulation and Formulation; CRC: FL, 2004.

(2) Rowe, R. C.; Sheskey, P. J.; Owen, S. C. Handbook of Excipients; Pharmaceutical Press: London, 2006.

(3) Sharma, V. K.; Kalonia, D. S. Polyethylene Glycol-Induced Precipitation of Interferon Alpha-2a Followed by Vacuum Drying: Development of a Novel Process for Obtaining a Dry, Stable Powder. AAPS PharmSci 2004, 6, 1-14.

(4) Nair, R.; Gonen, S.; Hoag, S. W. Influence of Polyethylene Glycol and Povidone on the Polymorphic Transformation and Solubility of Carbamazepine. Int. J. Pharm. 2002, 240, 11-22.

(5) Strickley, R. G. Solubilizing Excipients in Oral and Injectable Formulations. Pharm. Res. 2004, 21, 201-230.

(6) Rubino, J. T. Cosolvent and Cosolvency. In Encyclopedia of Pharmaceutical Technology; Marcel Dekker: New York, 1990.

(7) Spreti, N.; Germani, R.; Incani, A.; Savelli, G. Stabilization of Chloroperoxidase by Polyethylene Glycols in Aqueous Media: Kinetic Studies and Synthetic Applications. Biocatal. Bioreact. Prog. 2004, 20, 96-101.

(8) Jouyban, A. Review of the Cosolvency Models for Predicting Solubility of Drugs in Water-Cosolvent Mixtures. J. Pharm. Pharm. Sci. 2008, $11,32-58$.

(9) Jouyban, A. Solubility Prediction of Drugs in Water-Polyethylene Glycol 400 Mixtures Using Jouyban-Acree Model. Chem. Pharm. Bull. 2006, 54, 1561-1566. 
(10) Rytting, E.; Lentz, K. A.; Chen, X.-Q.; Qian, F.; Venkatesh, S. Aqueous and Cosolvent Solubility Data for Drug-Like Organic Compounds. AAPS J. 2005, 7, E78-E79.

(11) Jouyban, A.; Soltanpour, Sh.; Tamizi, E. Solubility Prediction of Solutes in Aqueous Mixtures of Ethylene Glycols. Pharmazie 2008, $63,548-550$

(12) Loftsson, T.; Hreinsdóittir, D. Determination of Aqueous Solubility by Heating and Equilibration: A Technical Note. AAPS PharmSciTech. 2006, 7, E29-E32.
(13) Shayanfar, A.; Acree, W. E., Jr.; Jouyban, A. Solubility of Lamotrigine, Diazepam, Clonazepam, and Phenobarbital in Propylene Glycol + Water Mixtures at 298. 15 K. J. Chem. Eng. Data 2009, 54, 11531157.

Received for review April 3, 2009. Accepted May 31, 2009.

JE900330P 